(C) 2007 ACM, Inc. Access to this work was provided by the University of Maryland, Baltimore County (UMBC) ScholarWorks@UMBC digital repository on the Maryland Shared Open Access (MD-SOAR) platform.

Please provide feedback

Please support the ScholarWorks@UMBC repository by emailing scholarworks-group@umbc.edu and telling us what having access to this work means to you and why it's important to you. Thank you. 


\title{
Towards Developing Assistive Haptic Feedback for Visually Impaired Internet Users
}

\author{
Ravi Kuber, Wai Yu and Graham McAllister \\ Queen's University Belfast \\ Belfast, BT7 1NN, UK \\ \{r.kuber,w.yu, g.mcallister\}@qub.ac.uk
}

\begin{abstract}
Haptic technologies are thought to have the potential to help blind individuals overcome the challenges experienced when accessing the Web. This paper proposes a structured participatory-based approach for developing targeted haptic sensations for purposes of web page exploration, and reports preliminary results showing how HTML elements can be represented through the use of force-feedback. Findings are then compared with mappings from previous studies, demonstrating the need for providing tailored haptic sensations for blind Internet users. This research aims to culminate in a framework, encompassing a vocabulary of haptic sensations with accompanying recommendations for designers to reference when developing inclusive web solutions.
\end{abstract}

\section{Author Keywords}

Blind, design methodology, haptic, participatory design, scenarios, Web accessibility.

\section{ACM Classification Keywords}

H5.2. Information interfaces and presentation (e.g., HCI): User Interfaces, Haptic I/O.

\section{INTRODUCTION}

Assistive technologies are commonly used by the blind community to access the Internet. Screen readers (e.g. JAWS [9], Window-Eyes [29]) and refreshable Braille output devices (e.g. Alva [2]) provide an audio or tactile representation of the data contained within a page. However, these tools are known to place restrictions upon the browsing process, by outputting information in a linear and time-consuming fashion. Non-textual content (e.g. images, tables, text formatting) is often key to gaining a full comprehension of a web page, but difficult to perceive using an assistive device. As a consequence, developing a mental model of the intended spatial layout can pose a challenge [31]. Improved non-visual presentation of a web interface would not only assist users in the exploration process, but could also help to improve the subjective browsing experience.

The sense of touch is a powerful modality for interface design. Haptic technologies have been shown to aid navigation through a virtual environment [27], assist the visualization of graphs [30], and enrich interaction for the user. Graphical User Interfaces (GUIs) have been haptically-augmented for both sighted and blind users, providing a tactual representation of icons, controls and windows, reinforcing the spatial metaphor of the desktop $[15,18,20,22]$. In the context of web browsing, haptic technologies have recently been developed to communicate the presence of HTML elements on an interface by using force-feedback [13], and tactile pin representations [24]. At present, there are no concrete guidelines for accessible haptic web interface design in existence.

As haptic technologies develop and low-cost devices become more widely available, designers are beginning to realize the advantages of using the sense of touch to create accessible browsing interfaces. However, the limited availability of both guidelines and structured haptic design methodologies, may lead developers to arbitrarily select sensations to map to HTML elements. Design choice could be based on nothing more than personal preference or ad hoc choice [21], influenced by the limited range of effects offered by current haptic technologies. Attempts could be made to either translate on-screen graphical icons into a non-visual format, or transfer mappings from other haptic interfaces, without considering the interaction techniques appropriate to non-visual presentation of a web interface and the additional assistance required by blind users when exploring a page. Poorly designed or selected haptic feedback would need to be explicitly learned, requiring time and effort to be invested by the blind user.

A framework housing a vocabulary of haptic sensations with accompanying guidelines for design, would act as a vital referencing tool, allowing developers to replicate feedback on their own web sites. It would serve to promote standardization, providing a safeguard against mappings varying from site-to-site. The tool would also support developers through the design and evaluation processes, 
hiding the unnecessary complexity often associated with haptic design.

In order to inform the framework, a structured design approach is required, to develop haptic sensations appropriate to the needs of blind individuals when accessing the Web. In this paper, we propose a five-step method, and report the preliminary haptic mappings to HTML elements resulting from application of the approach.

\section{RELATED WORK AND INTERFACE DESIGN}

Non-visual web interfaces have traditionally relied upon the use of audio to provide a representation of page contents [5, 7]. In terms of using haptic technologies, Roth et al. [25] have examined how blind participants interact with a touch sensitive tablet to access web-based content. Sounds represent the nature of the HTML tag touched, providing increased awareness of the position and meaning of each element. Auditory and tactile interfaces have been used by Asakawa et al. [3] to communicate structural information, such as groupings of elements. Tactile output has also been used by Rotard et al. [24], who have tactually represented non-textual information (e.g. tables, frames, colours and images), enabling blind individuals to gain an insight into the visual presentation of the page.

An assistive tool has been developed by our research group, consisting of a multimodal interface and content aware web plug-in [31]. The tool enables the user to explore web page layout through active haptic interaction (Figure 1). A forcefeedback mouse is used for navigating around the interface, and its cursor position is constantly monitored. Depending on the user's intention and the context of the task, appropriate haptic and auditory (speech and non-speech) prompts can be presented. These can include providing guidance to the desired destination, or informing users about the presence of elements located in close proximity to the cursor (e.g. images and hyperlinks) [31].

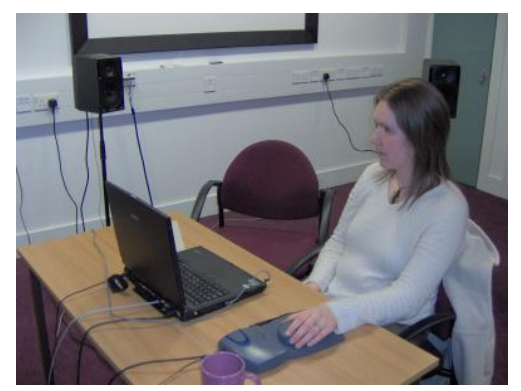

Figure 1: Blind user interacting with the multimodal interface

Evaluation of the interface revealed that haptic technologies played an influential role in enabling users to develop a mental representation of web page layout. However, it was thought that the haptic sensations mapped to HTML elements, could be improved to better support blind users when accessing the Web. For example, sensations mapped to hyperlinks should aim to ease the targeting process.
Additional elements (e.g. image-hyperlinks and form objects) would also need to be rendered, to provide a more representative view of the contents of a web page. In order to develop assistive sensations, a structured method is required. Resulting sensations can then be integrated with the assistive tool discussed by Yu et al. [31].

\section{Challenges for the Design Process}

'Top-down approaches' are often used when designing a haptic interface. The designer considers the need for providing a haptic representation, and finds an appropriate solution from a suite of technologies and methods. This often requires knowledge of those tools and their affordances [11]. However, such approaches can present a challenge for designers with a lesser amount of experience of developing haptic solutions. There is also the possibility that designers may create sensations without taking into consideration the needs of blind individuals when accessing a web page. James [8] has noted that blind users lack grounding in spatial and visual metaphors, which makes graphical interfaces difficult to access.

Participatory design is an approach that focuses on collaborating with intended users throughout the design and development process, rather than designing a system for "them" [6]. The process values interactive evaluation to gather and integrate feedback from intended users, thereby promoting design iteration. Participatory approaches have been used to develop assistive technology prototypes, adhering to the needs of the target groups $[6,14]$. However, even though the concept of haptic-based web exploration sounded appealing to blind people [16], the process of asking people with limited knowledge of haptic technologies to describe sensations for designing an interface can prove to be a challenge. The language of describing haptic phenomena is often limited to a small selection of words [28]. In order to conduct a participatory approach, blind users would need to first develop an awareness of haptic technologies to communicate design ideas. Similarly, haptic designers would need to understand the constraints imposed by assistive devices such as screen readers.

Developing haptic sensations that convey an underlying sense of meaning to the user are thought to provide assistive benefit. However, representing semantic information through the haptic channel is known to be a challenging process. The relatively narrow haptic bandwidth limits the number of distinct sensations which can be produced via current technologies. Designing haptic sensations using metaphor, suggestibility and simplicity could enhance interaction [11]. It is hoped that haptic sensations will aid the blind community when exploring web pages, where barriers to access are currently experienced. These mappings could also assist many blind individuals who are required to collaborate on web-based tasks with their fullysighted peers $[16,18]$. 
Pre-print: Kuber, R., Yu, W. \& McAllister, G. 2007: Towards Developing Assistive Haptic Feedback for Visually Impaired Internet Users. In proceedings of on Human Factors in Computing Systems - CHI'07, San Jose, USA, 1525-1534.

\section{METHODOLOGY SELECTED}

In an attempt to address the issues raised, a five-step method for developing targeted haptic feedback is proposed, as shown in Figure 2.

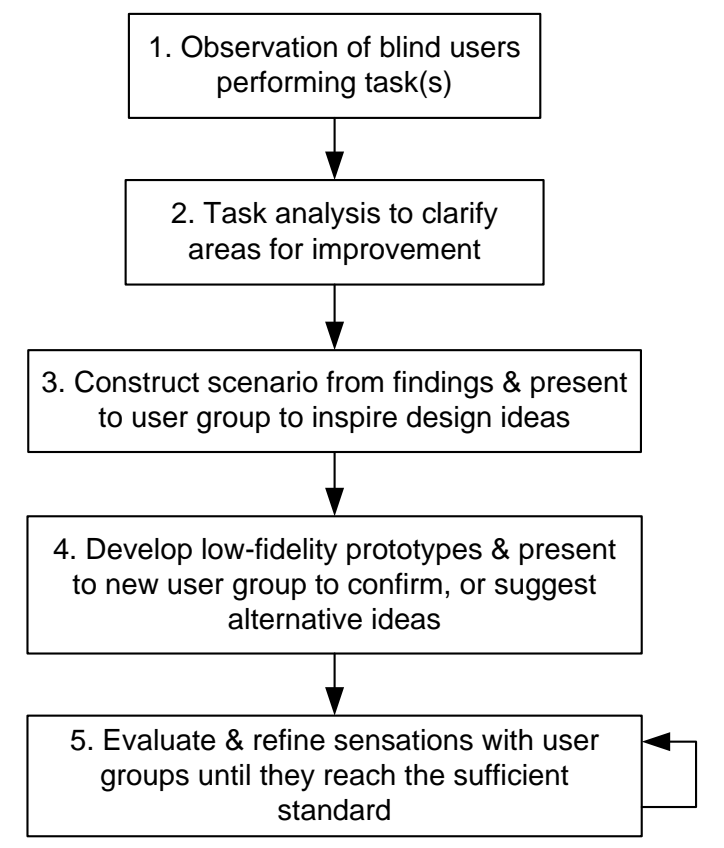

Figure 2: Five-step method to design haptic feedback

\section{Steps 1 \& 2: Observation and Task Analysis}

To observe blind people performing web-based tasks in conjunction with a screen reader, a series of web-based tasks are designed and presented to a sample of blind participants with varying levels of Internet experience. They are encouraged to "think aloud", verbalizing intended goals and outcomes of any actions made using the screen reader. The session is video recorded, and a hierarchical task analysis is performed on the footage. Tasks are decomposed into sub-tasks, and represented in the order which they are executed. The aim of the step is to analyse the textual or diagrammatic representations from the task analysis, to assess the challenges faced when using a screen reader, and to examine areas where additional assistance would be beneficial.

\section{Step 3: Scenario Design and Presentation}

Assemble a design team (also referred to as user group) consisting of haptic interface designers and blind screen reader users. Each participant is introduced to a number of haptic effects. The objective is for each participant to describe various sensations, and generate a set of descriptive terms pertaining to the sense of touch. These terms can be used for purposes of discussion when the scenario is presented.

On the basis of findings from the observation and task analysis steps, a rich story or scenario relating to haptic exploration of a web interface by a blind user can be designed. If findings from steps 1 and 2 have revealed that considerable time and mental effort is spent locating a certain element on a web page using a screen reader, this finding should be included in the scenario for purposes of discussion. The aim of presenting the scenario to the design team is two-fold. Firstly, participants can identify the role that haptic technologies can play on a web interface. It is possible that other modalities such as audio, could be more effective at communicating web-based information to a blind user. Secondly, if the haptic channel is thought to be appropriate for use, the session would allow for participants to brainstorm design ideas pertaining to the sense of touch.

The researcher should lead the group through the main issues covered by the scenario, allowing the participants to make design suggestions and reflect upon each others' ideas. Next, participants are requested to describe haptic sensations which could provide assistance to the blind user in the scenario. In order to suggest design ideas, participants should consider the purpose that elements (e.g. images and hyperlinks) serve on a web page, and how users currently interact with them. They should consider the special qualities that touch has to offer [11], and concepts such as metaphor. Sensations should not be considered in isolation. If participants suggest feedback to represent a particular page element, they should consider whether the sensation would work in conjunction with mappings defined for other elements.

Writing equipment and objects of various textures (props) should be provided as a method of externalising sensations, which participants may find difficult to describe. Resulting from design suggestions made, low-fidelity prototypes should be created using props, or by developing basic haptic effects with prototyping software, such as Immersion Studio (Figure 4) in conjunction with a force-feedback device.

\section{Step 4: Presenting to a New User Group}

Assemble a second user group comprising of blind screen reader users and haptic designers, who have not previously participated in a design session. The same scenario should be presented to the new group, coupled with the low fidelity prototypes suggested by the first user group. The aim of this step is for the user group to confirm the validity of design suggestions made by the first group, and to provide further feedback to strengthen the existing prototypes. Alternatively, they can suggest other ideas. These designs can again be mocked-up using props, or implemented through haptic prototyping software.

\section{Step 5: Further Design Refinement}

Assemble a design team consisting of participants from previous user groups, and present them with the same scenario, and prototypes resulting from steps 3 and 4 . This stage aims to iterate designs. By this stage, higher fidelity prototypes should be implemented and presented within web pages. It is envisaged that after a number of sessions, consensus on design for each mapping will be reached. If 
multiple representations are suggested, further sessions can be conducted to assess whether one of the sensations is more applicable within the context of the task. If a number of sensations are applicable, all representations are placed within the assistive framework, allowing the designer to test and select the most appropriate feedback for the context of use.

\section{APPLYING THE METHODOLOGY TO A CASE STUDY}

The case study that has been used refers to the process of web searching. Murphy et al. [16] have reported that this task is infrequently performed, as some blind users can find the process challenging. The reason that the task was selected was because it provided the opportunity to observe blind users attempting the search process, gaining an overview of search results, and the targeting of a particular result. It was hoped that any assistive sensations designed, would be of benefit to improving the search process, and provide support for general web page exploration.

\section{Steps 1 \& 2: Observation and Task Analysis}

Six blind individuals, aged between 18 and 32, with varying levels of experience with the Internet, were recruited for purposes of the study. All participants reported limited or absent functional vision, so used a screen reader to access the Web. Participants were presented with a training task, and then asked to perform a web search on the term "music technology" to locate a course related to the term. All participants used the JAWS screen reader which was customized to their own preferences. All participants opted to search using the Google engine (Figure 3). The majority of participants stated that they performed searches on a weekly basis, often to obtain reference materials for their studies, but on occasion to find out information about events, favorite bands, and radio programmes of interest.

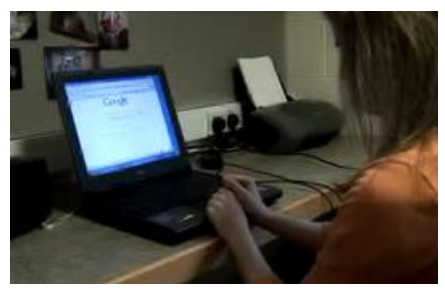

Figure 3: Blind participant performing web search task with screen reader

\section{Steps 3 - 5: Scenario Design and Presentation}

In order to facilitate discussion regarding design ideas, participants from each user group were introduced to twelve pairs of haptic effects beforehand. Effects include friction, damping, slopes, springs, waves and various forces, developed using Immersion Studio (Figure 4) played through the Logitech Wingman force-feedback mouse (Figure 5). Participants were asked to describe each pair of sensations, and were questioned on whether they could differentiate between paired stimuli.

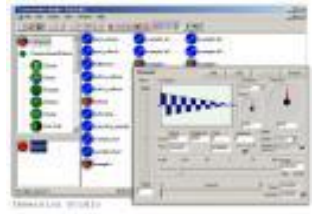

Figure 4: Immersion Studio application

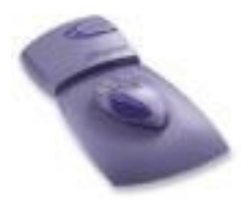

Figure 5: Logitech Wingman force-feedback mouse
The scenario reflected the experience of a congenitally blind student, Aidan - the actor or agent, who explored the Web using a multimodal browser with a haptic input/output device from his home - setting. The plot revolved around the actor whose goal was to perform a web search on an audio book title. An excerpt from the scenario is presented below.

"Aidan explores the web page using the haptic device, first moving past a large image, denoted by a haptic sensation. Directly below the image, Aidan perceives a search term box underneath his fingertips, and using the haptic device, checks that it is empty."

The researcher read out the scenario to each design team. Participants were then asked to discuss design ideas relating to each page element (e.g. images, hyperlinks, buttons), in turn. In addition, participants were asked how guidance could be assisted on a page, and how an overview of contents could be formulated. User groups were also invited to discuss whether haptic sensations could assist in the navigation process and aid in the development of an overview of contents.

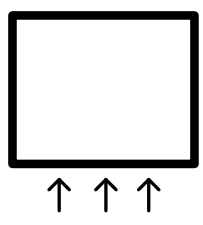

Figure 6: Haptic border marking the edges of an image

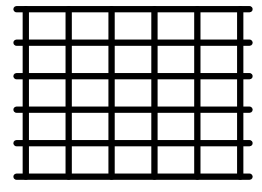

Figure 7: Spatial texture to provide awareness of the body of an element
Three user group sessions have been conducted. Four people took part in the first session; two haptic interface designers, one blind screen reader user, and an auditory interface designer. The auditory interface designer was asked to participate in the session, in order to suggest whether modalities other than haptics, could provide a more effective means of representing page-based information. Prototypes were developed resulting from the session. Examples can be seen in Figures 6 and 7.

The second user group session was conducted one month later. This group consisted of a blind haptic designer, a blind audio designer, and a fully sighted audio designer. The scenario was read out to the group with the addition of low fidelity prototypes developed from the findings of the first user group. Sensations were played to the user, as the scenario was read to the user group, as suggested by Pirhonen et al. [21]. An excerpt is shown: 
Pre-print: Kuber, R., Yu, W. \& McAllister, G. 2007: Towards Developing Assistive Haptic Feedback for Visually Impaired Internet Users. In proceedings of on Human Factors in Computing Systems - CHI'07, San Jose, USA, 1525-1534.

Once Aidan enters the search term, he tries to locate the Go button. He locates the haptic feedback representing a button located next to the search box <<play haptic sensation>>. If Aidan was less practised with the browser and device, the haptic device would have guided him to the centre of the button <<play haptic sensation >>.

The third user group session was conducted a month after the second group session, using the same members from the second team. Following a similar protocol, participants were introduced to the prototypes developed from both the first and second groups, to assess whether the iterated prototypes had matched their expectations. If not, feedback was requested on how to improve designs.

\section{RESULTS \& DISCUSSION}

\section{Steps 1 \& 2: Observation and Task Analysis}

The task analysis stage revealed that the screen reader was helpful to accomplish the search task, but did not provide all the support required by the users. Steps $1 \& 2$ helped to identify areas where additional feedback could be directed.

The majority of participants were experienced with using Google with the JAWS screen reader for purposes of conducting a web search. As a search box was present on the page, participants knew that they would need to use the screen reader's "forms mode" to enter a search term within the box. A keystroke was made to enter the "forms mode". Participants were noted to carefully listen to the corresponding auditory cue "Forms Mode on". After inputting the search term, participants would make another keystroke to exit the mode and continue with the search.

Strategies for gaining an overview of contents from the search results page varied from participant-to-participant. Some users would listen carefully to information regarding the title of the page, and the number of hyperlinks and headings present, to assess the amount of information that was contained within the page. Others would opt to move through a list of page hyperlinks to gain the gist. Both methods were noted to be time-consuming processes, and still did not provide an effective representation of page contents. It is possible that a shorter, more effective form of gaining an overview could provide more assistance when opening a page.

As the screen reader was known to output page information in sequential order, participants were aware they would be presented with links to other parts of the Google site and to sponsored links, before locating the main search results. Two participants had developed strategies to avoid listening to the extraneous information presented. By selecting the Page Down key a set number of times, they would be able to move past the sponsored links, closer towards the main search results. Other participants were noted to take time, moving through all the extraneous links to reach the main results. In this case, providing awareness of the location of refreshed content, or guidance towards it, could benefit blind users, as screen readers do not convey enough information regarding spatial layout.
Participants found that the main search results listed were often poorly labelled. It was difficult to isolate whether a search result relevant to the intended goal was present on the page. Some participants would be prepared to take the chance of selecting a result and then moving to the corresponding page to gain an overview. Others were prepared to spend more time than others using additional keystrokes to listen to the summary text associated with each hit. Figure 8 shows the breakdown of steps taken by one participant. Considerable effort was taken moving back and forth through each of the hits and corresponding summaries on just one search page, in order to isolate a relevant match. This was found to create a mild sense of frustration.

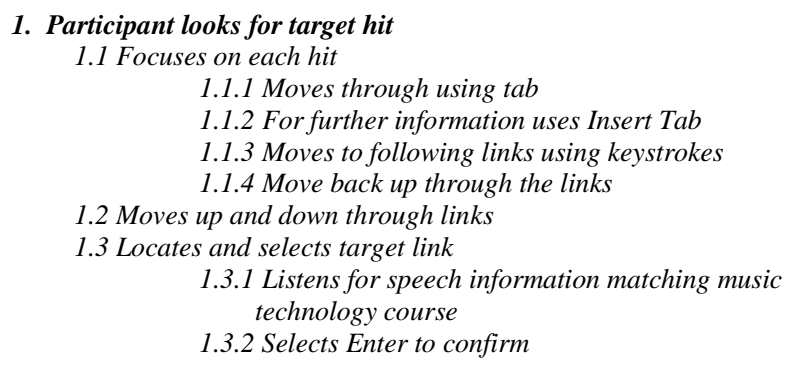

Figure 8: Excerpt from hierarchical task analysis

A less sequential method of exploring contents would benefit blind users, allowing them to gain a representation of the page.

\section{Step 3: User Group Session 1}

The sense of touch was thought by the group to be an effective medium to reference elements contained within a page. Haptic sensations could provide "landmarks" or "anchors", enabling users to develop a mental map of the page layout. It was thought that haptic technologies could be used to assist in attracting a user to a particular area of a page, and provide a barrier to avoid areas of a web page completely. This would benefit the user when encountering extraneous information, such as adverts or repetitive links.

Participants were more reticent about using haptic feedback to produce an overview of a web page. Modalities such as audio were thought to be more effective at conveying this information succinctly. One of the haptic interface designers suggested by moving the mouse over hapticallyrendered elements on a page, an overview of contents could be formed. For example, the user could perceive if the page was densely packed with elements such as images and hyperlinks, or contained mainly text. The user would be able to able to make a decision whether to stay on the page, or to return when more time was available. Ideally, sensations would need to be short in duration, perceivable and non-intrusive, similar to the design of non-visual icons $[4,12]$. It was thought that the user should have control over the number of elements that are haptically-represented within the interface, and should also have the option of additional navigational assistance. 
Participants were able to suggest recommendations arising from the use of metaphor. Assistive navigation could be represented by the idea of "holding and guiding a child's hand" to guide the user to a particular part of a web page. This was described as a firm but non-intrusive sensation, which would guide the user between two points on the page. It was thought that the user should be able to override the assistive navigation, if desired.

A magnetic-style effect was recommended to represent a hyperlink. This effect would allow the user to hover over the hyperlink, without the risk of accidentally moving away from the target. The idea was welcomed by the blind panelist, who stated that as a keyboard was her sole input device, she may lack the ability to make the fine movements needed for mouse use. The group knew from the pre-task stage of the existence of spring effects in the Immersion Studio application, allowing the user to be attracted to or repelled from a certain position. This was thought to present a starting point for developing a sensation, which could be tested by the group.

\section{Step 4: User Group Session 2}

The participants in the second user group were generally able to strengthen ideas suggested by the first user group, by providing suggestions on how to improve the existing prototypes. They were also able to suggest new creative ideas for design, if they felt that previous suggestions were not appropriate.

Participants found the magnetic-style effects suggested by the first group for representing hyperlinks and imagehyperlinks to be meaningful. They were able to build on the suggestions, by explaining how these two elements could be differentiated from one another. Instead of providing an additional sensation to represent each element, they recommended using different types of magnetic effect. For example, hyperlinks could be represented by "providing the sensation of being attracted into the centre of a long thin channel or river", as they were thought to be visually long and thin, and are known to take time to listen to using a screen reader. Image-hyperlinks could be represented by "providing a sensation of being attracted into the centre of a funnel". By providing the sensation of a cardboard-style texture or a vibration when hovering over the body of an image-hyperlink similar to that of an image, users would be able to group image-related items. This process of grouping was thought to aid interpretation.

In contrast to findings of the first group, the second user group recommended not to add any additional haptic sensations to provide awareness of the page background. The group feared that if additional effects were mapped to the background, sensations representing elements already present on the page (e.g. mappings for images and hyperlinks) could be difficult to perceive. Providing a border around a page was thought to provide enough feedback to make the user aware that he/she would be hovering over a web page. Using the metaphor of a picture frame, it was thought that the user would find it difficult to leave the main body of the page (interior of the picture), and would have to move out of the page by climbing the walls on to the border (frame of the picture). Additional feedback could be encased around the border, providing awareness when moving outside the confines of the border, on to the desktop. Participants remarked that the sensation experienced around the border should feel pleasant to touch. MacLean [11] states that the gratuitous addition of nicefeeling qualities can often enhance the pleasure of interaction.

\section{Step 5: User Group Session 3}

The third user group was able to assess prototypes developed as a result of the first and second groups. In response to issues concerning hyperlinks, the group found that multi-directional spring effects were adequate to represent both hyperlinks and image-hyperlinks. The spatial texture effect overlaying the body of the imagehyperlink would allow participants to differentiate between the two elements (Figures 9 and 10). Participants generally preferred the ideas of utilizing spatial texture effects to represent elements such as the area outside the border and image-related items, rather than relying on the use of vibrations. The group thought that the vibration effects tested, would be more appropriate for providing an alert to the presence of an unwanted object (e.g. for extraneous information such as an advert or Flash object).
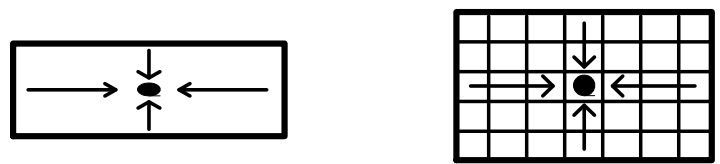

\section{Figure 9: Multi-dimensional spring attracting user to centre of hyperlink}

\begin{abstract}
Figure 10: Multi-dimensional spring with spatial texture attracting user to the centre of the image-hyperlink
\end{abstract}

The user group was introduced to a lowered enclosure effect designed to represent a search box. The effect enabled the user to gently descend into the interior of the search box to explore its contents. A subtle spatial texture was developed to represent the presence of text inside the box. If the user wanted to enter information, a small periodic wave was played to slightly nudge the user, providing awareness that he/she had clicked within the confines of the search box and data could now be entered. The group continued with suggestions for refinement. They recommended increasing the strength of the spatial texture inside the search box, to ensure that all participants would be able to perceive textual content within the box. If the sensation was too weak, there was the danger that the element would be occluded when haptically-scanned. 
Pre-print: Kuber, R., Yu, W. \& McAllister, G. 2007: Towards Developing Assistive Haptic Feedback for Visually Impaired Internet Users. In proceedings of on Human Factors in Computing Systems - CHI'07, San Jose, USA, 1525-1534.

\section{Presentation of Findings}

Preliminary findings from the iterations conducted are summarized and presented in Appendix 1. These sensations are compared with mappings suggested for general haptic user interfaces. Results of the comparison have indicated while some parallels can be drawn between sensations which are applicable for both general user interfaces and web interfaces, using a structured design approach has led to the development of targeted assistive feedback suitable for the needs of blind users when performing a web-based search, and for general exploration of the Web. It is thought that findings from our study will assist designers in the development of inclusive web browsing solutions.

\section{Comparison with Mappings from Prior Studies}

Similarities are seen for representing certain elements such as buttons. Rosenberg \& Brave [23] have recommended using attractive feedback to help users navigate towards an element. Results from the five-step approach also revealed that attractive feedback would also be useful on a web page. However, unlike in previous studies, haptic designers and blind users were able to test the sensations offering the benefits of their experience to tailor them to the needs of users. One haptic designer knew from previous experience, that using inappropriately sized magnetic (spring) effects could potentially lead to users overshooting their targets. Participants were introduced to a web page containing four image-hyperlinks, which were located in close proximity to one another. Participants were asked to explore the page to assess whether the spring effects mapped to imagehyperlinks were appropriately sized or whether they needed to be refined. The iterative process ensured that effects were not too strong, and that sensations were perceivable and distinctive from other feedback present on a page.

Miller \& Zelesnik [15] have recommended creating a slight ridge around icons to make a dimpled effect, making them easier to target. Findings from the user groups have revealed that even though the process of raising borders around elements such as images was found to be beneficial for purposes of developing spatial awareness, it could cause confusion if the mouse cursor was displaced when encountering a ridge. The user group recommended representing an image as a lowered enclosure, allowing the user to move into the body of the image. As images are larger than icons on a page, the development of a texture would provide information about the location of the body of an image. By moving over the sensations mapped to images, participants stated that they could not only spatially map the position of images, but were also able to roughly estimate image sizes.

Some findings from the five-step approach differed from past studies. Ramstein et al. [22] have discussed use of a gutter, allowing the user to highlight the corners and edges of the window. O'Modhrain \& Gillespie [20] have represented windows edges as grooves, providing awareness of location. Findings from the five-step approach suggested that grooves were more useful for attracting a user to a certain region of the page. Using our approach, it was found that participants preferred the idea of a raised border effect, which would allow the user to locate his/her position within a web interface. The user would need to take more effort to leave the interior of the page but would not be constrained to a particular section. An additional outer boundary spatial texture would provide non-intrusive feedback allowing the user to navigate to his/her area of choice.

O'Modhrain \& Gillespie [20] have recommended representing a checkbox as a border with a repelling block at one end which becomes an attracting spring when the checkbox is checked. Spring effects were recommended by participants in the five-step approach, in order to attract the user to the relatively small surface area of a checkbox, whether checked or not. Experiencing the prototype encouraged further discussion. Participants suggested that a vibration could be appropriate to convey awareness of the active checkbox. Grouping checkboxes in close spatial proximity was also thought to provide benefit. This could be done within an enclosure or 'trough', allowing the user could perceive the walls of the area where all checkboxes are located.

Adelstein \& Rosen [1] have recommended using a negligible amount of friction, to make the user aware that he/she is navigating within the environment. However, findings from our work indicate that additional haptic sensations applied to the page background were considered unnecessary. As web pages are often densely packed with information, rendering a separate effect to represent the background was thought to overload the user.

The sensations presented in Appendix 1 have aimed to represent a small selection of elements present on a web page. It has been acknowledged that for the development of a vocabulary, further mappings will need to be added. It is thought that the same approach could be employed, with a view to developing these sensations. The five-step method could also be used to examine more complex browsing issues such as the resizing and movement of browser windows, or scrolling through a lengthy page.

\section{General Issues Related to Approach}

\section{Developing a Common Language}

Introducing the participants to pairs of haptic sensations allowed screen reader users and haptic designers to communicate using a shared set of terms. Words such as "resistant, smooth, tough, attractive, textured etc" were used to convey design ideas. Blind screen reader users became more "technology-aware" during this stage, providing them with an idea of the capabilities and constraints of working with haptic technologies. If participants were unable to describe a design idea, writing paper or props also provided a useful means of communication with other members of the group. 


\section{Participatory Design Approach}

The participatory approach was thought to be integral to the design process. Haptic designers were able to work together with the blind screen reader users as partners, to consider interaction design for non-visual exploration of a page, and suggest ideas which could be discussed and tested. Both parties could bring the benefits of their collective experiences to each session, and learn from each other. Blind participants could discuss additional limitations posed by screen readers, and their use of touch for purposes of communication (e.g. Braille) and for differentiating between objects (e.g. surface textures, weight, temperature). Haptic designers were able to highlight sensations which they had developed for other interfaces in the past, and discuss the constraints associated with using a haptic device.

In the participatory design process, it is important to remember that end users are not expert designers. Users may make design suggestions, but these may not necessarily be appropriate or feasible for use on an interface. Testing should take place to ensure that suggestions fit their purpose, and work in conjunction with mappings to other elements. In our study, sensations were usually iterated multiple times within each session to reach the required standard.

\section{Administering Scenarios to User Groups}

A fairly detailed scenario was presented to each user group, which appeared to inspire ideas for design. Participants were generally able to identify with the blind character in the story, and his attempt to perform a web-based search using a haptic device.

In order to enable all members of the design team to discuss ideas in more detail, group size was reduced from four participants to three. A set amount of time was allocated to discuss each mapping, to avoid the sessions from overrunning. However, even with fewer participants, the process of introducing haptic sensations to the whole group proved to be a difficult process. The researcher needed to interrupt the flow of discussion, to play suggested haptic sensations to each participant and provide assistance to blind users when interacting with the force-feedback mouse. To address similar challenges, a pre-recorded scenario with embedded effects has been recommended to coordinate the presentation of auditory sensations to a user group [17]. However, this approach would not overcome the practical challenges associated with introducing participants to haptic sensations simultaneously. Additional helpers would be required to ensure that all members of the group can interact with each individual haptic device to experience cues. Participants are then free to evaluate sensations and provide feedback on each others' thoughts.

The scenario itself could have produced more appropriate ideas for design by considering the environmental context in greater detail. Pirhonen et al. [21] have suggested providing more information about the physical, social and psychological context. The first user group suggested that further consideration should be made regarding use of the keyboard within the scenario. A blind user may have to continuously move his/her hands away from the device to type or to perform keystroke commands, and then locate the haptic device to perceive further sensations. Provision would need to be made if the cursor was displaced as a result.

\section{Prototyping}

Although prototyping has been recognized as an efficient means of developing user interfaces, the debate rages whether prototypes need to be complete, realistic, or reusable to be effective [26]. Low-fidelity prototypes could be quickly mocked-up using paper, props or developed using the Immersion Studio application, to provide participants a general idea of how the user would aim to interact with the tactile sensations suggested. However, there are concerns raised over the applicability of lowfidelity prototypes to real-world applications. Higher fidelity prototypes were developed to provide a more realistic view of how sensations would be presented when exploring a web interface. Effects were coded through the use of Javascript, and accessed through either static HTML pages or dynamically through the use of the multimodal interface described.

\section{Validation of the Framework}

In order to ensure that sensations are refined to a sufficient standard, designs can be evaluated against the same scenario, and against additional scenarios exploring other contexts of use (e.g. when gaining general or in-depth knowledge from a page, or when filling out a web-based form). In order to assess the benefit brought by assistive sensations, it seems pertinent to observe blind users exploring the Web using mappings from the framework. An interim evaluation has been conducted using sensations resulting from the second user group session [10]. Findings have shown that that blind participants were able to differentiate between the mappings on a page, developing a more comprehensive mental representation of spatial layout. Participants were generally able to locate and select targets within short periods of time. A rich and stimulating browsing experience was also created.

\section{CONCLUSION \& FURTHER WORK}

This paper has proposed a structured approach to design assistive haptic feedback for use when exploring the Web, and has presented results from the application of the approach. Findings have been compared with mappings from other haptic interfaces, showing the need for tailored design. As a next step in the research project, it appears pertinent to refine and rigorously test mappings. We also aim to extend the vocabulary of elements, and develop a set of guidelines for applying these sensations to a web interface. 
Pre-print: Kuber, R., Yu, W. \& McAllister, G. 2007: Towards Developing Assistive Haptic Feedback for Visually Impaired Internet Users. In proceedings of on Human Factors in Computing Systems - CHI'07, San Jose, USA, 1525-1534.

\section{ACKNOWLEDGMENTS}

We wish to thank Emma Murphy, Sile O'Modhrain, Philip Strain and Samantha Chui-Chui Tan for their help and advice. This work is supported by Eduserv.

\section{REFERENCES}

1. Adelstein, B.D. and Rosen, M.J. Design and Implementation of a Force Reflecting Manipulandum for Manual Control Research. ASME Winter Annual Meeting, (1992), 1-12.

2. Alva Braille Display. http://www.visioncue.com/products_01.asp.

3. Asakawa, C., Takagi, H., Ino, S. and Ifukube, T. Auditory and Tactile Interfaces for Representing the Visual Effects on the Web. Proc. Assets, ACM Press (2002), 65.-72.

4. Brewster, S.A. and Brown, L.M. Tactons: Structured Tactile Messages for Non-Visual Information Display. Proc. Australian User Interface Conference, (2004), 1523.

5. Donker, H., Klante, P. and Gorny, P. The Design of Auditory User Interfaces for Blind Users. Proc Nordichi, ACM Press (2002), 149-156

6. Ellis, R. D. and Kurniawan, S. Increasing the Usability of Online Information for Older Users: A Case Study in Participatory Design. International Journal of HumanComputer Interaction, 12, 2 (2000), 263-276.

7. IBM Home Page Reader 3.04. http://www.ibm.com/able/solution_offerings/hpr.html

8. James, F. Presenting HTML Structure in Audio: User Satisfaction with Audio Hypertext. Proc. ICAD, (1996).

9. JAWS For Windows. http://www.freedomscientific.com/.

10.Kuber, R., Murphy, E., McAllister, G., Strain, P. and $\mathrm{Yu}, \mathrm{W}$. Evaluation of an Assistive Web Interface: A Collaborative Approach. Internal Technical Report, (2007).

11. MacLean, K. E. Designing with Haptic Feedback. Proc. ICRA, (2000).

12. MacLean, K. and Enriquez, M. Perceptual Design of Haptic Icons. Proc. Eurohaptics, (2003), 351-363.

13.Magnusson, C., Tan, C. and Yu, W. 2006. Haptic Access to 3D Objects on the Web. Proc. Eurohaptics (2006).

14.Massimo, M. and Baeker, R. Participatory Design Process with Older Users. Proc. Ubicomp Workshop, (2006).

15.Miller, T. and Zelesnik, R. An Insidious Haptic Invasion: Adding Force Feedback to the X Desktop. Proc UIST, (1998), 59-64.
16.Murphy, E., Kuber, R., McAllister, G., Strain, P. and $\mathrm{Yu}, \mathrm{W}$. An Empirical Investigation into the Difficulties Experienced by Visually Impaired Internet Users. To appear in Universal Access in the Information Society, (2007).

17.Murphy, E., Pirhonen, A., McAllister, G., Yu W., A Semiotic Approach to the Design of Non-Speech Sounds. Proc. Haptic Audio Interaction Design Workshop, (2006), 121-132.

18.Mynatt, E. and Weber, G. Nonvisual Presentation of Graphical User Interfaces: Contrasting Two Approaches. Proc. CHI, ACM Press (1994), 166-172.

19.Oakley, I., Adams, A., Brewster, S. and Gray, P. Guidelines for the Design of Haptic Widgets. Proc. BCS HCI, (2002), 195-212.

20.O'Modhrain, M.S. and Gillespie, B. The Moose: A Haptic User Interface for Blind Persons. Proc. Third WWW6 Conference, (1997).

21.Pirhonen, A., Murphy, E., McAllister, G. and Yu, W. Non-Speech Sounds as Elements of a Use Scenario: A Semiotic Perspective. Proc. ICAD, (2006), 134-140.

22.Ramstein C., Martial O., Dufresne A., Carignan M., Chasse P. and Mabilleau P. Touching and Hearing GUI's: Design Issues for the PC-Access System. Proc Assets ACM Press (1996), 2-9.

23.Rosenberg, L. and Brave, S. Using Force Feedback to Enhance Human Performance in Graphical User Interfaces. Proc. CHI, ACM Press (1996), 291-292.

24.Rotard, M., Knodler, S. and Ertl, T. A Tactile Web Browser for the Visually Disabled. Proc Hypertext and Hypermedia, ACM Press (2005), 15-22.

25.Roth, P. Petrucci, L.S., Assimacopoulos, A. \& Pun, T. Audio-Haptic Internet Browser and Associated Tools for Blind Users and Visually Impaired Computer Users. Proc. Workshop on Friendly Exchanging Through the Net, (2000), 57-62.

26.Rudd, J., Stern, K. \& Isensee, S. Low vs High Fidelity Prototyping Debate. Interactions, 3, 1 (1996), 76-85.

27.Sjostrom, C. Designing Haptic Computer Interfaces for Blind People. Proc. ISSPA, (2001).

28.Sullivan, C. and Chang, A. An Activity Classification for Vibrotactile Phenomena. Proc. Haptic and Audio Interaction Design Workshop, (2006), 145-156.

29. Window-Eyes 6.0. http://www.gwmicro.com/Window-Eyes/.

30.Yu, W. and Brewster, S.A. Evaluation of Multimodal Graphs for Blind People. Universal Access in the Information Society 2, 2 (2003), 105-124.

31.Yu, W., Kuber, R., Murphy, E., Strain, P. and McAllister, G. A Novel Multimodal Interface for Improving Visually Impaired People's Web Accessibility. Virtual Reality 9, (2006), 133-148. 


\section{Appendix 1: Comparison of our Findings with Previous Studies}

\begin{tabular}{|c|c|c|}
\hline Elements & Findings from Five-Step Approach & \multirow{4}{*}{$\begin{array}{l}\text { Other Studies for General Interfaces } \\
\text { To convey position of icons, Miller \& } \\
\text { Zeleznik [15] have recommended making a } \\
\text { slight ridge around images, providing a } \\
\text { dimpled effect. Ramstein et al. [22] and } \\
\text { O'Modhrain \& Gillespie [20] have also } \\
\text { recommended that the user can perceive } \\
\text { boundaries. } \\
\text { In order to attract the user (to click on an } \\
\text { element), Ramstein et al. [22] have suggested } \\
\text { that the more depth an object has, the more the } \\
\text { pointer is attracted to it and the user is } \\
\text { attracted to the area. With sufficiently high } \\
\text { levels of attraction, small objects are easier to } \\
\text { select [22]. } \\
\text { Menus have been rendered by Miller \& } \\
\text { Zeleznik [15] by ridges in between items. } \\
\text { Oakley et al. [19] has also examined the idea } \\
\text { of targeting. Widgets augmented with } \\
\text { attractive basins or haptically walled areas are } \\
\text { thought be beneficial but consideration should } \\
\text { be made over the object's shape and the likely } \\
\text { path taken by the user over it. }\end{array}$} \\
\hline Images & $\begin{array}{l}\text { A slightly lowered or raised enclosure effect should be used to encase the visual border } \\
\text { of the image. A weak spatial texture should be applied to the image's interior. } \\
\text { When moving through a set of thumbnails, an enclosure effect should be placed on } \\
\text { three sides of the image, to produce an effect akin to 'falling down a set of steps'. } \\
\text { No feedback should be applied to spacer or decorative images. }\end{array}$ & \\
\hline $\begin{array}{l}\text { Image- } \\
\text { hyperlinks }\end{array}$ & $\begin{array}{l}\text { A multi-dimensional spring effect should be used to direct the user towards the relative } \\
\text { centre of an image-hyperlink. } \\
\text { An optional weak spatial texture effect (the same texture applied to images) can be } \\
\text { mapped to an image-hyperlink, to provide awareness of the element. } \\
\text { If there are many image-links located in close spatial proximity, the designer may need } \\
\text { to adjust strength of effects. }\end{array}$ & \\
\hline Hyperlinks & $\begin{array}{l}\text { A multi-dimensional spring effect should be used to direct the user towards the relative } \\
\text { centre of a hyperlink. Optional use of distinctive spatial texture or weak periodic wave } \\
\text { effect to alert presence of a hyperlink. } \\
\text { Other more subtle effects, such as visited links or links to PDFs, can be presented } \\
\text { through audio. } \\
\text { To represent a list of links, an effect should be applied enabling the user to feel like } \\
\text { he/she is running his/her finger through the inner fold (crease) of a restaurant menu. }\end{array}$ & \\
\hline $\begin{array}{l}\text { Page } \\
\text { border }\end{array}$ & $\begin{array}{l}\text { The user should not be constrained within the web page window. A lowered enclosure } \\
\text { effect should be used to provide a border around the HTML content. This would allow } \\
\text { the user to explore the page, and perceive its boundaries. To leave the page, the user } \\
\text { should be able to push-out on to the raised border. } \\
\text { A non-intrusive strong spatial texture effect should be placed around the border, to } \\
\text { provide awareness of position on the interface. }\end{array}$ & $\begin{array}{l}\text { To provide awareness of borders on a window, } \\
\text { Ramstein et al. [22] have recommended a } \\
\text { physical gutter surrounding each window. } \\
\text { Users can follow the rectangular boundary, } \\
\text { feeling a difference when they reach a corner. } \\
\text { Miller \& Zeleznik [15] have placed ridges } \\
\text { around windows, making it harder to select the } \\
\text { Window Manager's decoration border. A } \\
\text { groove can be placed around window, } \\
\text { providing additional awareness [20]. }\end{array}$ \\
\hline $\begin{array}{c}\text { Page } \\
\text { background }\end{array}$ & $\begin{array}{l}\text { No haptic sensation should be used to represent the background, as the other mappings } \\
\text { on the page should stand out. The location of text on a web page should not be } \\
\text { communicated through haptic technologies. }\end{array}$ & $\begin{array}{l}\text { Miller \& Zeleznik [15] have provided } \\
\text { additional awareness of the workspace by } \\
\text { using the metaphor of a shallow box. Bottom } \\
\text { of the box is the primary interaction surface. } \\
\text { Adelstein \& Rosen [1] have recommended the } \\
\text { use of friction to convey presence within the } \\
\text { virtual environment. }\end{array}$ \\
\hline $\begin{array}{l}\text { Search box } \\
\& \text { button }\end{array}$ & $\begin{array}{l}\text { A lowered enclosure effect should be applied to a search box. This would allow the } \\
\text { user to move into a box and explore its contents. A strong spatial texture effect should } \\
\text { be used to convey the presence of text within a search box. To indicate that the user } \\
\text { has clicked inside a box, a weak periodic wave can be used to provide a slight nudge. } \\
\text { To represent a button, a multi-dimensional spring effect should be used to attract the } \\
\text { user to the centre of the object. No further sensation is required to represent the body of } \\
\text { a button. }\end{array}$ & $\begin{array}{l}\text { Boxed objects are represented are thought to } \\
\text { make it easy for users to locate and select } \\
\text { objects [22]. } \\
\text { Rosenberg \& Brave [23] used attractive force- } \\
\text { fields to attract users to a button. Also } \\
\text { recommended by Oakley [19] to attract users } \\
\text { to a widget. }\end{array}$ \\
\hline Checkboxes & $\begin{array}{l}\text { A spring effect should be used to attract users to a checkbox. A vibration can convey } \\
\text { the presence of an active checkbox. All related checkboxes should be grouped in an } \\
\text { enclosure or 'trough'. }\end{array}$ & $\begin{array}{l}\text { A checkbox is represented as a border with a } \\
\text { repelling block at one end, which becomes an } \\
\text { attracting spring when the checkbox is } \\
\text { checked [20]. }\end{array}$ \\
\hline $\begin{array}{l}\text { Locational } \\
\quad \text { effect } \\
\text { towards an } \\
\text { object on a } \\
\quad \text { page }\end{array}$ & $\begin{array}{l}\text { A periodic wave should be used which increases in strength as the user moves towards } \\
\text { the element. This effect would be beneficial when attempting to locate one particular } \\
\text { element (e.g. text box or hyperlink) on a page containing a small number of objects. }\end{array}$ & $\begin{array}{l}\text { No specific advice given. Researchers have } \\
\text { recommended the use of attractive basins [19]. } \\
\text { Sjostrom [27] recommends making well } \\
\text { defined reference points to ease navigation, }\end{array}$ \\
\hline $\begin{array}{c}\text { Assistive } \\
\text { navigation }\end{array}$ & $\begin{array}{l}\text { A haptic groove or gentle directed motion towards the target element should be used to } \\
\text { assist the navigation process. }\end{array}$ & \\
\hline
\end{tabular}

